\title{
PARTITIONING OF COOMASSIE BRILLIANT BLUE INTO DMAEMA CONTAINING POLY(HEMA)-BASED HYDROGELS
}

Christian N. Kotanen ${ }^{1}$, Dileep R. Janagam ${ }^{2}$, Rachelle Idziak ${ }^{1}$, Luke Rhym ${ }^{1}$, Ryan Sullivan ${ }^{1}$, Ann M.

Wilson $^{3,4}$, Tao L. Lowe ${ }^{2}$ and Anthony Guiseppi-Elie ${ }^{1,4, *}$

${ }^{1}$ Center for Bioelectronics, Biosensors and Biochips (C3B), Clemson University, Advanced Materials Research Center, Anderson, South Carolina 29625, USA.

2

${ }^{2}$ Department of Pharmaceutical Sciences, University of Tennessee Health Science Center, Memphis, Tennessee 38163, USA

${ }^{7}$ Department of Chemistry, University of the West Indies, St. Augustine, Trinidad and Tobago

${ }^{4}$ ABTECH Scientific, Inc., 800 East Leigh Street, Richmond, Virginia 23219, USA

*Correspondence should be addressed to Anthony Guiseppi-Elie

Phone: +1-864-656-1712

Fax: +1-864-656-1713

E-mail: guiseppi@clemson.edu 


\section{ABSTRACT}

The loading and release of anti-inflammatory drugs from hydrogel-coated implantable devices is a demonstrated approach in mitigating the inflammatory response of implantable devices. Poly(2hydroxyethyl methacrylate)-based hydrogels possessing ionizable 2-(dimethylamino)ethyl methacrylate (DMAEMA) and cross-linked with varying concentrations (1, 3, 5, 7, 9 and 12 mol\%) tetra(ethylene glycol) diacrylate (TEGDA) were synthesized and studied for their degree of hydration, glass transition temperature and partitioning of zwitterionic Coomassie Brilliant Blue (CBB), a zwitterionic drug surrogate known to bind to amines. Using UV-Vis spectroscopy, the partition coefficient of CBB within the hydrogels was found to average $6.69 \pm 0.12$ but to rise monotonically from 6.54 (1 mol \%) to 6.84 $(12 \mathrm{~mol} \%)$ as a function of TEGDA mol\% or cross-link density in accord with a monotonic reduction of the degree of hydration. The absence of DMAEMA was found to reduce the partition coefficient of CBB from 6.5 to 4.1. Both formulations, with and without DMAEMA (pKa=7.5), showed an independence of $\mathrm{pH}$ over the physiologically relevant range, $\mathrm{pH} 5-\mathrm{pH} 9$, confirming that this difference was not due to Henderson-Hasselbalch ionization of the pendant group. The presence of DMAEMA resulted in a 1.44 fold increase in CBB loading confirming complex formation. The absence of DMAEMA still resulted in considerable partitioning of $\mathrm{CBB}$ into the hydrogel suggesting that the affinity between $\mathrm{CBB}$ and DMAEMA may not be the only relationship controlling the partition coefficient.

Keywords: hydrogels, poly(HEMA), hydration, Coomassie blue, drug loading, partitioning, glass transition temperature 


\section{INTRODUCTION}

Hydrogels are polymeric materials containing hydrophilic and hydrophobic constituents that aid in closely controlling physicochemical properties. Poly(2-hydroxyethyl methacrylate) or [p(HEMA)] is an inherently biocompatible, hydrophilic hydrogel whose synthesis readily allows incorporation of additional monomers or pre-polymers containing unique functional groups for use in novel industrial formulations and biomedical applications [1-4]. Applications for $\mathrm{p}($ HEMA) include wear resistant hydrophilic surface coatings for prosthetics [5], antimicrobial wound dressings [6], intramuscular biosensor coatings [7], tissue engineering scaffolds [8], generation of bone-like materials [9], imparting hemocompatibility to metal surfaces [10], improving hardness and elasticity of brittle substrates [11], and soft contact lenses [12], and sophisticated drug delivery systems [13-15]. When conferred with pH and/or biologically derived responsiveness, poly(HEMA)-based hydrogels have been used in responsive drug delivery with engineered feedback control [14, 16-20]. Mechanical properties and hydration characteristics of $\mathrm{p}(\mathrm{HEMA})$ are typically controlled through bifunctional crosslinking agents such as oligo- and poly- ethyleneglycol diacrylates e.g. tetra(ethylene glycol) diacrylate (TEGDA), which, when added at varying concentrations, influence the modulus and the void volume through the cross-link density of the hydrogel $[14,21]$. However, such crosslinks may in addition be virtual and thus arise from hydrogen bonding [22, 23], electrostatic interactions[24], polyplex formation [25] and molecular physical entanglements. Moreover, these hydrogels may be readily molecularly engineered to contain a wide variety of pendant and crosslinking bioactive moieties that render them biologically responsive wherein the response of the hydrogel is triggered by recognition of a biological agent conferred by an immobilized biorecognition species. In previous work, we have shown the incorporation of 2(dimethylamino)ethyl methacrylate (DMAEMA) for the construction of pH-responsive hydrogel microforms that released insulin upon exposure to glucose. Within that construct, $\mathrm{pH}$ changes induced by the generation of gluconic acid $\left(p K_{a}=3.68\right)$ by immobilized glucose oxidase (GOx) caused 
protonation of the pendant tertiary amine $\left(p K_{a}=7.5\right)[26]$ to induce swelling of the hydrogel matrix. Increased hydration resulted in an increase in the diffusion coefficient, and hence release rate, of insulin that had been also loaded into the hydrogel construct. The result was a form of all synthetic artificial pancreas responsive to external glucose levels [15]. A recent in silico demonstration of a Gen-3 selfregulated delivery of drug with feedback control from poly(HEMA)-based hydrogels was made by Guiseppi-Elie et al. [27] and an in vivo demonstration of a Gen-3 hydrogel system was reported by Gordijo et al. (2011), wherein blood-glucose concentrations were controlled by a glucose-responsive, insulin-releasing hydrogel [28].

Coomassie Brilliant Blue (CBB) is a zwitterionic molecule often used to dye proteins in gel electrophoresis that can also be used to visualize penetrance of liquids into hydrogels [29]. An equilibrium measure of this penetrance is known as the partition coefficient. This phenomenon is observed when solutes in a solution diffuse into a hydrogel despite concentration gradients, resulting in a higher concentration of the solute within the hydrogel than within the solution. Measuring the partition coefficient of $\mathrm{CBB}$ into poly(HEMA)-based hydrogels lends itself to application in drug and biomolecule loading. Negatively charged biomolecules having potential applications in bioactive p(HEMA) hydrogels include cell adhesive factors [30], heparin [31], DNA aptamers [32], plasmids [33], and antimicrobial peptides $[34,35]$. The primary objective of this work was to explore the drug loading potential of novel, biomimetic p(HEMA)-based hydrogels by determination of the partitioning coefficient using $\mathrm{CBB}$ as a drug surrogate. A combination of external and internal factors is responsible for influencing and controlling partitioning into hydrogel systems. External factors include temperature, $\mathrm{pH}$ and ionic strength whereas internal factors include gel hydrophilicity, viscosity, mechanical strength, surface charge, crosslink density and degree of hydration [36]. In realizing the partitioning capabilities of the hydrogel, the potential for loading drugs with similar molecular structure may be better understood. The effect of crosslink density of hydrogels on partitioning of CBB was explored using 
TEGDA as a crosslinker. It is understood that increased mole fraction of TEGDA within a hydrogel will confer more cross-linkages and therefore produce a more rigid structure $[37,38]$. We hypothesize that to a first approximation, network pore size or hydrogel void volume is associated with drug sorption capacity and diffusion capabilities. However, in the presence of specific interactions (e.g. electrostatic interactions), this may be altered. Thus major functional constituents of a formulated biomimetic hydrogel, such as zwitterionic phosphorylcholine, may alter drug loading capacity. Consequently, this works also examined the role of cationogenic DMAEMA in the partitioning of CBB.

There are four important parameters for the structural characterization of swollen hydrogels and from which bulk and transport properties may be derived. These are i) the swelling factor, Q, ii) the degree or percentage hydration, $\mathrm{DoH}$, iii) the average molecular weight between cross-links, $M_{c}$, and iv) the network pore size, $\xi$. The parameter $M_{c}$ represents the molecular weight of polymer chains between neighbouring cross-links or junctions. The network mesh size or pore size, $\xi$, is determined by the average distance between crosslink points in the hydrogel and thereby controls the degree of hydrogel porosity. Figure 1 presents a 2-D rendering that schematically illustrates the concept of swelling of a hydrogel by the imbibition of water and serves to schematically illustrate $M_{c}$ and $\xi$ in the 2-D network.

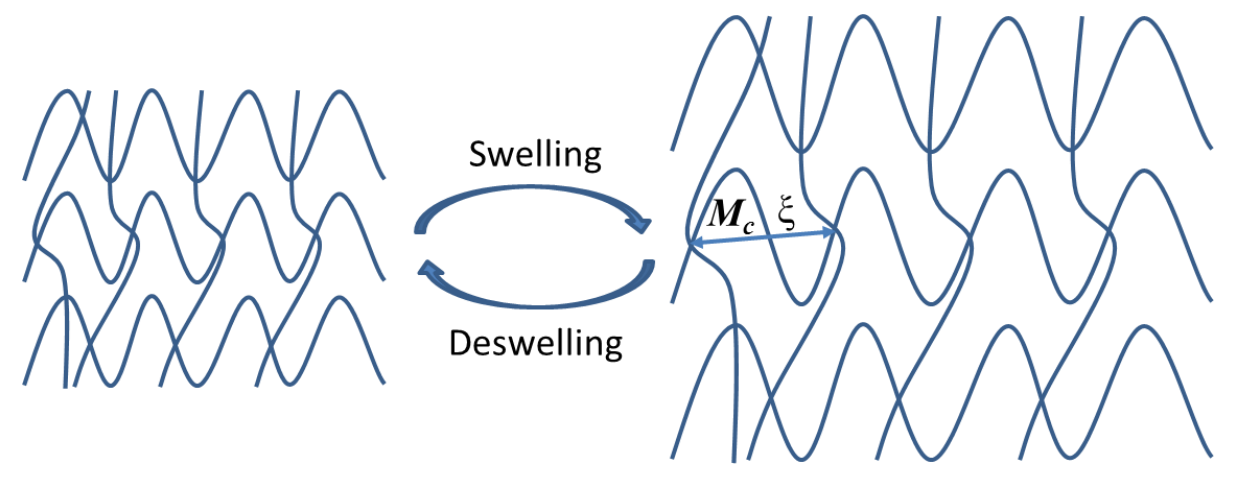

Figure 1. A 2-D rendering that schematically illustrates the concept of swelling of a hydrogel by the imbibition of water and schematically illustrates $M_{c}$ and $\xi$ in the 2-D network.

The swelling factor, $\mathrm{Q}$, represents the ratio of the volume of swollen gel: 
$Q=\frac{V_{s}}{V_{p}}=\frac{1}{\vartheta_{2, s}}$

Where $V_{s}$ is the volume of the swollen hydrogel, $V_{p}$ is the volume of the polymer and $\vartheta_{2, s}$ is the volume fraction of the polymer. The degree of hydration, $\mathrm{DoH}$, expressed as a percentage, represents the ratio of the amount of water taken up by the hydrogel to the total weight of the hydrated hydrogel. The $D o H$ allows calculation of the void fraction, $\varepsilon$.

Degree of hydration $(\mathrm{wt} \%)=\left(\frac{M_{H G}-M_{D G}}{M_{H G}}\right) 100 \%$

$\varepsilon=\left(\frac{M_{H G}-M_{D G}}{\rho_{\text {water }}}\right) /\left(\frac{M_{H G}}{\rho_{H G}}\right)$

Where $M_{H G}$ is the weight of the hydrated hydrogel at equilibration, $M_{D G}$ is the weight of the dry hydrogel, $M_{H G}-M_{D G}$ is the equilibrium water content $(E W C)$ of the hydrogel, and $\rho_{w a t e r}$ and $\rho_{H G}$ are the densities of water and the hydrated hydrogel, respectively. Clearly, to a first approximation and in the absence of virtual crosslinks and polyplex formation [25], highly cross-linked polymer networks will exhibit lower swelling than loosely cross-linked networks and thus have smaller swelling factors, smaller molecular weights between crosslinks, lower degrees of hydration, and lower porosity or void fraction. With respect to their pore size and pore size distribution, hydrogels may be divided into macroporous, microporous or nonporous architectures. The swelling factor and degree of hydration can both be determined experimentally by swelling measurements and allows the further calculation of $M_{c}$ and pore size.

The partition coefficient, $\Phi$, is a measure of the distribution of a molecule between contacting immiscible media. The molecule distributes itself between the two media according to its chemical compatibility and at a rate depending upon its mobility within the two media. Often the media are of the same phase, e.g., liquid/liquid as shown in Figure $2 \mathbf{A}$ in which case it is called a liquid-liquid 
extraction when the molecule moves from being predominantly in one medium to being predominantly in the other. Sometimes the media are pseudo-solid/liquid as shown in Figure $\mathbf{2} \mathbf{B}$ in which case it is called absorption or sorption. In chemical engineering, absorption is a physicochemical process whereby atoms, ions or molecules leave the gaseous or liquid phase and enter a liquid or bulk solid phase. It is an important unit operation. The physicochemical process of absorption is, for simple solutes and polymer systems, governed by Henry’s Law and Fick's Law of diffusion. Henry's Law describes the solubility of the solute within the contacting solid phase and Fick's laws describe the movement of the solute within the polymer phase. However, these simple idealized treatments are often accompanied by interactions between the solute and the pseudo-solid that give rise to complex formation that alters partitioning, distribution and polymer architecture [39, 40].

Specifically, the partition coefficient, $\Phi$, is the ratio of the molar concentrations of the solute in the two contacting but immiscible phases at equilibrium. For a hydrogel system:

$\Phi=\frac{\left[C_{g e l}\right]}{\left[C_{\text {soln }}\right]}$

Where $\Phi$ is the partition or distribution coefficient, $\left[C_{g e l}\right]$ is the molar concentration of the solute within the hydrogel and $\left[C_{\text {soln }}\right]$ is the molar concentration of the solute within the solution. The approach adopted in this work was to measure the optical density of the CBB in a known volume of bathing solution for a prescribed volume of hydrogel microform of known crosslink density and composition at a controlled temperature. The system was then allowed to achieve equilibrium and OD measurements were repeated to constancy. From a mass balance, the concentration of CBB within the hydrogel microform was calculated. The partition coefficient was then taken as the ratio of the concentrations under those test conditions. We are concerned with three key questions; the influence of the crosslink density on $\Phi$, the concentration dependence of $\Phi$, and the influence of the presence of ionic groups on $\Phi$. 

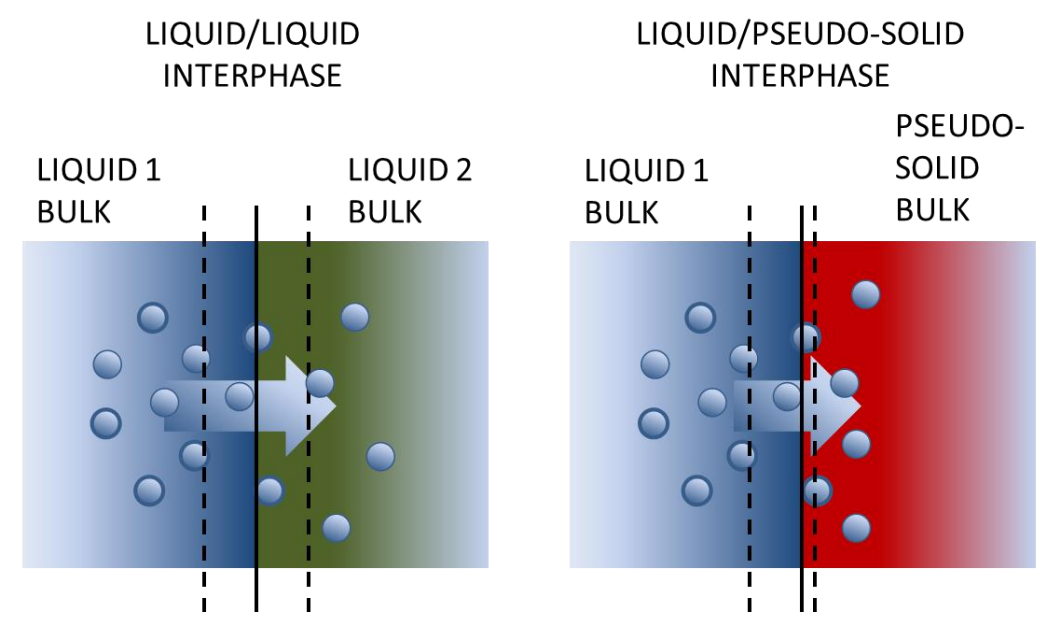

Figure 2. Schematic illustration of the transport of molecular constituents from one phase to another; A. From Liquid 1 to immiscible Liquid 2. B. From Liquid 1 to pseudo-solid wherein molecule-medium complexation phenomena may influence distribution. 


\section{EXPERIMENTAL}

\subsection{Chemicals and reagents}

Octadecyltrichlorosilane (OTS), Coomassie Brilliant Blue dye (CBB), 2-hydroxyethyl methacrylate (HEMA), tetra(ethylene glycol) diacrylate (TEGDA, technical grade), oligo(ethylene glycol)(400)methacrylate (OEG(400)MA), $N$-[Tris(hydroxymethyl)methyl]acrylamide (HMMA, 93\%), polyvinylpyrrolidone (pNVP, $\mathrm{M}_{\mathrm{w}} \sim 1,300,000$ ), 2-(Dimethylamino)ethyl methacrylate (DMAEMA, 98\%), the photo-initiator 2,2-Dimethoxy-2-phenylacetophenone (DMPA, 99+\%), and all other common solvents and buffers were purchased from Sigma Aldrich Co. (St. Louis, MO, USA). HEPES buffer was prepared at $0.025 \mathrm{M}$ and $\mathrm{pH}=7.35$. The HEMA, methacrylate and diacrylate reagents were passed over an inhibitor removal column (Sigma-Aldrich) for removal of the polymerization inhibitors hydroquinone and monomethyl ether hydroquinone before using them in the preparation of the hydrogel cocktail. Solutions were prepared in deionized water prepared by purifying distilled water through a Milli-Q${ }^{\circledR}$ plus (Millipore Inc.) ultrapure water system.

\subsection{Materials and methods}

Glass microscope slides (Gold Seal, Thermo Scientific) were surface modified and rendered hydrophobic by treatment with OTS for use in the preparation of hydrogel discs. Slides were first placed into a UV-ozone cleaner (Boekel Industries) and irradiated for 5 minutes on each side followed by a 1 minute ultrasonication in isopropyl alcohol (IPA). Plasma modification of the slides to generate hydroxyl groups onto the silicon dioxide was performed under vacuum in the presence of small amounts of water vapor using a Harrick Plasma Cleaner (Harrick Plasma). Following plasma activation, microscope slide surfaces were silanized by incubation in a $0.1 \mathrm{wt} \%$ solution of OTS in toluene for 45 minutes followed by ultrasonication in IPA for 5 minutes to remove excess silanol. Curing of the silanol was then performed using sequential 20 minute intervals of $40{ }^{\circ} \mathrm{C}, 110{ }^{\circ} \mathrm{C}$ and $40{ }^{\circ} \mathrm{C}$. Hydrophobic microscope slides were stored dry under argon until use. Hydrogel monomer cocktails of HEMA, 
TEGDA, OEG(400)MA, HMMA, pNVP, DMAEMA and DMPA were prepared according to the mole percentages found in Table 1 in a $20 \%$ v/v solvent of 1:1 water:ethylene glycol. The resulting structures are schematically illustrated in Figure 3.

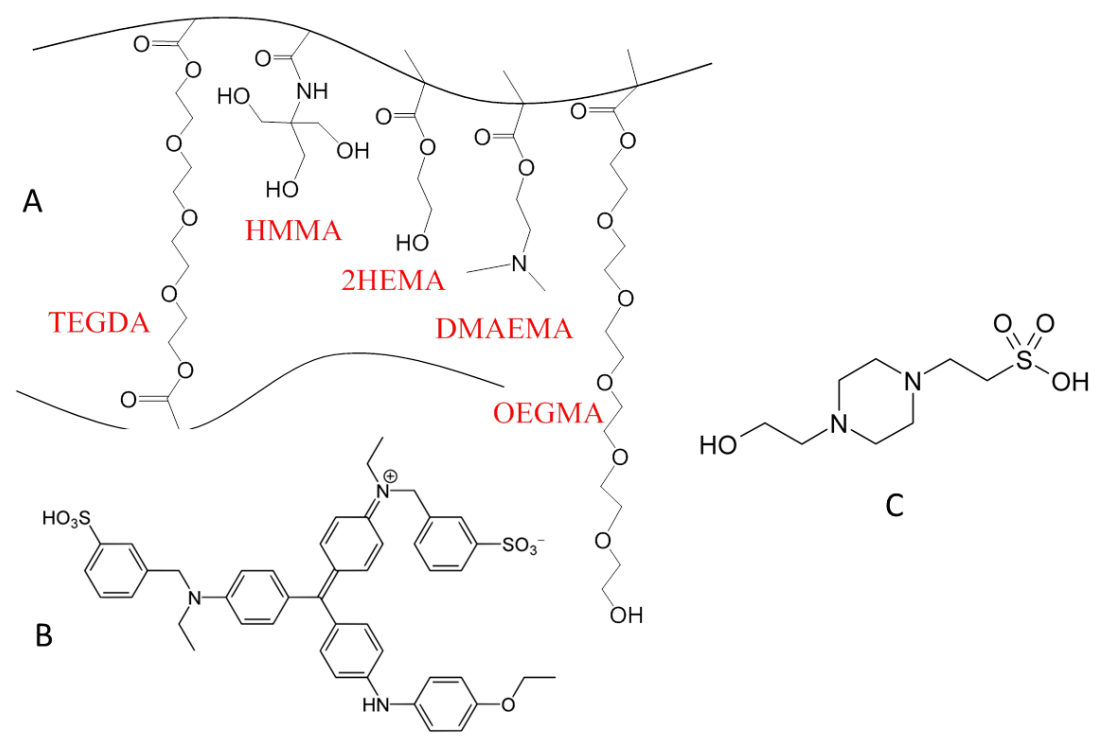

Figure 3. A. Schematic illustration of the polymerized molecular constituents of the bioactive hydrogel. B. Structure of CBB. C. Structure of HEPES (4-(2-hydroxyethyl)-1-piperazineethanesulfonic acid)

Table 1. Monomer cocktail formulations for the preparation of varying mol\% TEGDA crosslinked poly(HEMA)-based hydrogels containing DMAEMA (left) and no DMAEMA (right) prepared in triplicate.

\begin{tabular}{|l|c|c|c|c|c|c|c|c|c|c|c|c|}
\hline $\begin{array}{l}\text { Crosslink density } \\
\text { (mol\%) }\end{array}$ & \multicolumn{2}{|c|}{1} & \multicolumn{2}{|c|}{3} & \multicolumn{2}{|c|}{5} & \multicolumn{2}{c|}{7} & \multicolumn{2}{c|}{9} & \multicolumn{2}{c|}{12} \\
\hline HEMA & 81 & 86 & 79 & 84 & 77 & 82 & 75 & 80 & 73 & 78 & 70 & 75 \\
\hline TEGDA & 1 & 1 & 3 & 3 & 5 & 5 & 7 & 7 & 9 & 9 & 12 & 12 \\
\hline OEG(400)MA (n=10) & 5 & 5 & 5 & 5 & 5 & 5 & 5 & 5 & 5 & 5 & 5 & 5 \\
\hline HMMA & 5 & 5 & 5 & 5 & 5 & 5 & 5 & 5 & 5 & 5 & 5 & 5 \\
\hline PNVP & 2 & 2 & 2 & 2 & 2 & 2 & 2 & 2 & 2 & 2 & 2 & 2 \\
\hline DMAEMA & 5 & 0 & 5 & 0 & 5 & 0 & 5 & 0 & 5 & 0 & 5 & 0 \\
\hline
\end{tabular}




\section{DMPA}

\begin{tabular}{|l|l|l|}
\hline 1 & 1 & 1 \\
\hline
\end{tabular}

1

\begin{tabular}{l|l|l|}
1 & 1 \\
\hline
\end{tabular}

\begin{tabular}{l|l}
1 & 1 \\
\hline
\end{tabular}

\begin{tabular}{l|l|l|l|l|l|}
1 & 1 & 1 & 1 \\
\hline
\end{tabular}

1

1

\subsection{Preparation and conditioning of hydrogel dises}

Silicone isolators (664206, Grace Biolabs) of $4.5 \mathrm{~mm}$ diameter by $1.0 \mathrm{~mm}$ depth were pressuresealed onto hydrophobic microscope slides and the individual chambers were filled with the monomer cocktail until gel had formed a meniscus over the isolator $(\sim 30 \mu \mathrm{L})$. Isolators filled with monomer cocktail were covered with a second hydrophobic slide, sandwiching the liquid hydrogel. The assemblies were placed in the UV Crosslinker (CX-2000, UVP, Upland, CA, USA) and irradiated for 5 min at a wavelength of $366 \mathrm{~nm}\left(2.3 \mathrm{~W} / \mathrm{cm}^{2}\right)$ under flowing nitrogen. Hydrogels of all crosslink densities (1-12 mol\%) were prepared in this fashion. Crosslinked hydrogel discs were removed from the silicone molds and unreacted monomer was extracted by sequential incubation in 100\% EtOH and EtOH:HEPES buffer of 75:25, 50:50, 25:75 v\% EtOH:HEPES or EtOH:DI $\mathrm{H}_{2} \mathrm{O}$ of 75:25, 50:50, 25:75 v\% EtOH:DI $\mathrm{H}_{2} \mathrm{O}$ at $37^{\circ} \mathrm{C}$ for $1 \mathrm{~h}$ each depending upon the final need. Finally, the hydrogel samples were placed in $100 \%$ HEPES buffer or DI $\mathrm{H}_{2} \mathrm{O}$. This procedure was used to solvent extract unreacted monomer and hydrate the hydrogel at a mild rate to an extent governed by the network density and buffer ionic strength.

\subsection{Characterization of hydrogels}

\subsubsection{Degree of hydration}

The degree of hydration (DoH) of the hydrogels containing 1-12 mol\% TEGDA crosslinker with and without 5 mol\% DMAEMA in DI water at RT $\left(22{ }^{\circ} \mathrm{C}\right)$ was determined by gravimetric analysis. Firstly, following overnight equilibration and the removal of surface excess water with lint-free paper (Kimwipes ${ }^{\circledR}$ ), the weight of the fully hydrated hydrogels (HG) equilibrated in DI water was measured using an analytical balance $( \pm 0.1 \mathrm{mg})$. Hydrogel samples were then dried to constant weight using Freeze Dry System (Freezone 4.5, Labconco). The samples were further kept in the $-80{ }^{\circ} \mathrm{C}$ freezer for $12 \mathrm{~h}$ then allowed to dry in the lyophilizer for 2 days at a pressure of $100 \mathrm{mtorr}$ and $-80{ }^{\circ} \mathrm{C}$ and the 
weight repeatedly measured to constant weight. Equation 2 was used to calculate the percentage hydration.

\subsubsection{Water states in hydrogels}

The water states in the hydrated hydrogels containing 3 mol\% TEGDA crosslinker with and without 5 mol\% DMAEMA were measured and calculated using a differential scanning calorimeter (DSC) (Q2000, TA Instruments) equipped with a low-temperature cooling module and using, the method previously reported with slight modification [19-21]. Briefly, the hydrated hydrogels in water and HEPES buffer with known $\mathrm{DoH}$ were surface wiped, weighed and sealed in individual hermetically sealed aluminium pans (Tzero hermetic lid, 901684.901; Tzero pan, 901683.901). Samples were equilibrated at $-40{ }^{\circ} \mathrm{C}$ and then heated up to $30{ }^{\circ} \mathrm{C}$ at a rate of $10{ }^{\circ} \mathrm{C} / \mathrm{min}$ under constant purging of nitrogen at $50 \mathrm{~mL} / \mathrm{min}$. For each measurement, five samples were analysed. The enthalpy of the endotherm, $\Delta H_{\mathrm{m}}$, at $0{ }^{\circ} \mathrm{C}$ was obtained by integration of the endothermic peak using TA Universal analysis software and normalized with respect to the weight of the hydrated gel with a unit of $\mathrm{J} / \mathrm{g}$ of hydrated gel. The deionized water was used as a reference and the melting enthalpies for freezing free water and freezing bound water were assumed to be the same as that of bulk water $\left(\Delta H_{0}=334 \mathrm{~J} / \mathrm{g}\right)$. Small differences in the enthalpies of the melting ice of different crystals were neglected. The ratio of the $\Delta H_{\mathrm{m}} / \Delta H_{0}$ represents grams of freezable water per gram of the swollen hydrogel as shown in equation 5.

$\frac{W_{\mathrm{f}}}{M_{\mathrm{HG}}}(\mathrm{wt} \%)=\frac{\Delta H_{\mathrm{m}}}{\Delta H_{0}}$

The amount of non- freezing bound water per gram of hydrated gel was taken as the difference between the $\mathrm{DoH}$ and the amount of freezing water per gram of hydrated gel using equation 6.

$\frac{W_{\mathrm{nfb}}}{M_{\mathrm{HG}}}(\mathrm{wt} \%)=D o H-\frac{W_{\mathrm{f}}}{M_{\mathrm{HG}}}$ 


\subsubsection{Glass transition temperature $\left(T_{\mathrm{g}}\right)$ of hydrogels}

$\mathrm{Tg}$ of the hydrogels containing $3 \mathrm{~mol} \%$ TEGDA crosslinker with and without $5 \mathrm{~mol} \%$ DMAEMA was determined by thermal analysis using a DSC Q2000 (TA Instruments). Around 7 to 10 mg of dried hydrogel was cut, placed into individual hermetically sealed aluminium pans (Tzero hermetic lid, 901684.901; Tzero pan, 901683.901). Each sample was equilibrated at $-20{ }^{\circ} \mathrm{C}$ and run for two consecutive cycles at a heating rate of $10{ }^{\circ} \mathrm{C} / \mathrm{min}$ from $-20{ }^{\circ} \mathrm{C}$ to $200{ }^{\circ} \mathrm{C}$. The first heat cycle will assess the hydrogels' properties in the as-molded condition, and will erase the thermal history of the hydrogels. The second heat cycle will evaluate the inherent properties of the hydrogels. Three different drying methods were employed: Hydrogel samples were vacuum (23 inHg) dried at room temperature, vacuum dried (23 inHg) at $80^{\circ} \mathrm{C}$, and freeze dried for overnight prior to the $T_{\mathrm{g}}$ measurements.

\subsection{Partitioning of Coomassie Brilliant Blue into hydrogels}

Solutions of eight different concentrations of CBB $(0.1,0.5,1,2,3,4,5,6 \mathrm{wt} \%)$ were prepared in HEPES buffer $(0.025 \mathrm{M}, \mathrm{pH} 7.35)$. Aliquots of $300 \mu \mathrm{L}$ ( 10 times the volume of each hydrogel disc) of each solution were transferred to each of 3 rows of a 96-well plate. A ninth column of 3 wells was filled with 100\% HEPES buffer to serve as control. This was repeated 6 times, one for each crosslink density of the hydrogel. The filled $96-$ well plate was warmed to $37{ }^{\circ} \mathrm{C}$ with gentle shaking. The absorbance of CBB at $550 \mathrm{~nm}$ was measured using the Synergy Mx Monochromator-Based Multi-Mode Microplate Reader (BioTek Instruments, Inc., Winooski, VT USA). Absorbance spectroscopy was used for quantification of $\mathrm{CBB}$ partitioning into hydrogels. A calibration curve of $\mathrm{CBB}$ in HEPES buffer solution was separately created and used to convert absorbance units to concentration. The difference in absorbance between the buffer solution before the hydrogels were equilibrated and after equilibration was indicative of the amount of $\mathrm{CBB}$ that had successfully partitioned into the hydrogel. Following incubation in $100 \%$ HEPES buffer, one disc was transferred to each of the wells containing $0,0.1,0.5,1$, 2, 3, 4, 5, $6 \mathrm{wt} \% \mathrm{CBB}$ in HEPES. Hydrogels were then allowed to equilibrate overnight in a $37^{\circ} \mathrm{C}$ water 
bath after which they were removed (with care, to ensure minimum loss of liquid from each plate) and the plates read once more using the Synergy Mx Microplate Reader. The partition coefficient $(\Phi)$ of CBB between HEPES buffer and the biomimetic hydrogels were calculated from the change using equations 7 and 4:

$C_{\text {total }}=\frac{m_{1} \vartheta_{1}}{V_{\text {total }}}=\frac{C_{\text {gel }} V_{\text {gel }}+C_{\text {soln }} V_{\text {soln }}}{V_{\text {gel }}+V_{\text {soln }}}$

Technical replicates allowed the coefficients to be examined for their statistical validity. The volume of the gel $V_{\text {gel }}$ was calculated using previously established hydrogel swelling properties in HEPES buffer [41]. The concentration of $\mathrm{CBB}$ partitioned into the hydrogel was estimated by subtracting the initial absorbance concentration of $\mathrm{CBB}$ in the HEPES solutions from the absorbance measured after equilibration of the hydrogel discs.

\section{6. $\mathrm{pH}$ dependence of the partition coefficient of CBB into hydrogels}

The $\mathrm{pH}$ dependence of $\mathrm{CBB}$ partitioning was evaluated by formulating a second batch of hydrogels at a fixed 3 mol\% crosslink density that was formulated with and without DMAEMA using the same procedure outlined above (Table 1). $\mathrm{CBB}$ solutions were $\mathrm{pH}$ adjusted using $\mathrm{HCl}$ or $\mathrm{NaOH}$ to create five different solutions of HEPES $(\mathrm{pH}=5.0,6.0,7.0,8.0$, and 9.0), each with 3 wt\% CBB. An aliquot of each pH HEPES solution was left un-dyed to serve as a control. Changes in volume due to the exclusion of DMAEMA in the hydrogel network were measured and accounted for in equations 1 and 2. Using this information, the partition coefficients for the hydrogels with and without DMAEMA and for $\mathrm{pH}$ adjusted solutions were measured.

\section{RESULTS AND DISCUSSION}

The presence of the hydrophobic but potentially ionisable $3^{\circ}$ amine, DMAEMA, as a co-monomer at $5 \mathrm{~mol} \%$ is expected to alter the partitioning of ionized Coomassie Brilliant Blue into the 
poly(HEMA)-based hydrogel. Such change, reflected in the partition coefficient, may arise because of the altered hydration characteristics of the hydrogel that is co-incident to the chemical modification of the backbone, or to the result of changing segmental dynamics that alter the absorption of the model drug, or due to chemical interactions between the added co-monomer and the model drug. To evaluate these possible influences, the physico-chemical characteristics of the hydrogels were measured and were compared with the measured partition coefficient.

\subsection{Hydration and states of water within hydrogels}

The transport (rate) and sorption (capacity) of drugs into hydrogel nano-and micro-forms [27] are expected to be strongly influenced by the amount of water and the distribution of this water among the many possible states within the hydrogel [42]. The $D o H$ and void fraction, $\varepsilon$, as a function of cross link density were calculated using equations 2 and 3, respectively. Figure 4 shows the gravimetrically determined $\mathrm{DoH}$ expressed as a percentage and measured for the two hydrogel systems, $0 \mathrm{~mol} \%$ DMAEMA and 5 mol\% DMAEMA, both as a function of cross-linker concentration over the range corresponding to $1-12$ mol\% TEGDA at $37^{\circ} \mathrm{C}$. This graph shows the results of triplicate measures of two technical replicates and thus each point is represented by $n=6$. Figure $4 \mathrm{~A}$ presents the results obtained in DI water and Figure $4 \mathbf{B}$ presents the results obtained in $\mathrm{pH}$ 7.35 HEPES buffer. The presence of 5 mol\% DMAEMA dramatically altered the hydration characteristics of the p(HEMA)based hydrogels, greatly reducing their degree of hydration. In the absence of DMAEMA the hydrogels demonstrated a monotonic decrease in percentage hydration from $46 \%$ to $31 \%$ with the increase of 1 mol\% to 12 mol\% TEGDA. With 5 mol\% DMAEMA, the hydrogels were less hydrated and became invariant relative to their respective DMAEMA-free counterpart up to 7 mol\% TEGDA. However, at 9 and $12 \mathrm{~mol} \%$ TEGDA, the presence of DMAEMA was associated with increased hydration relative to the DMAEMA-free counterpart and these two points followed the same downward trend as the 
DMAEMA-free hydrogels. There was only a modest lowering of the overall degree of hydration when studied in HEPES buffer and the trends as a function of mol\% TEGDA were quite similar.

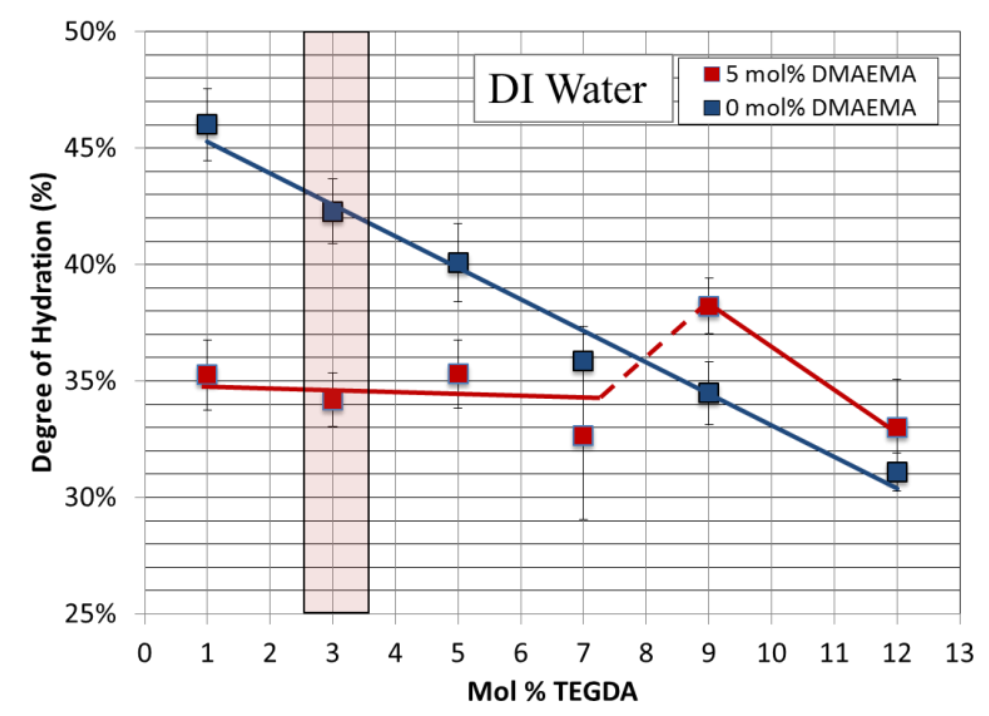

Figure 4A. The gravimetrically determined degree of hydration (\%) of the p(HEMA)-based hydrogels measured in DI water at $37^{\circ} \mathrm{C}$ as a function of mol $\%$ TEGDA cross-linker when the hydrogel contains $0 \mathrm{~mol} \%$ or $5 \mathrm{~mol} \%$ DMAEMA $(n=6)$.

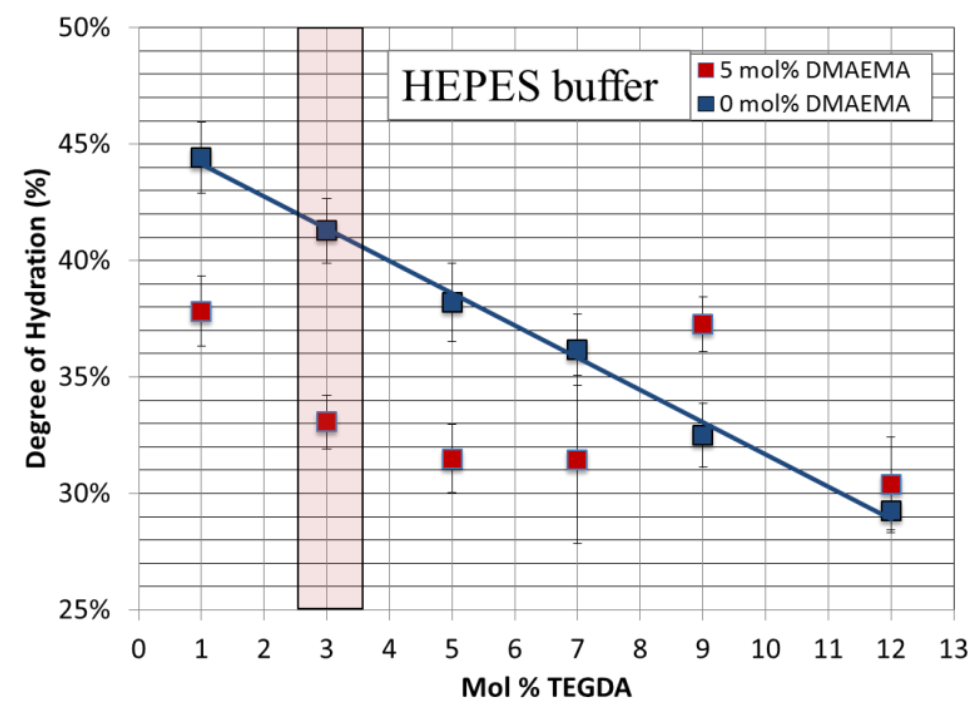

Figure 4B. The gravimetrically determined degree of hydration (\%) of the p(HEMA)-based hydrogels measured in HEPES buffer at $37^{\circ} \mathrm{C}$ as a function of mol \% TEGDA cross-linker when the hydrogel contains $0 \mathrm{~mol} \%$ or $5 \mathrm{~mol} \% \operatorname{DMAEMA}(n=6)$. 
To more deeply investigate the distribution of water states within these hydrogels, the $3 \mathrm{~mol} \%$ TEGDA crosslinker formulation possessing $0 \mathrm{~mol} \%$ or $5 \mathrm{~mol} \%$ DMAEMA was studied by DSC following equilibration in DI water or HEPES buffer. Water present in hydrated hydrogels is known to be present in three conditions, i) freezable free water $\left(W_{\mathrm{ff}}\right)$, ii) freezable bound water $\left(W_{\mathrm{fb}}\right)$ (freezing point depressed), and iii) and non-freezable bound water $\left(W_{\mathrm{nfb}}\right)$ [19]. The freezable free water $W_{\mathrm{ff}}$ does not participate in any interaction with the polymer chains while the freezable bound $W_{\mathrm{fb}}$ water slightly interacts with the polymer chains. The sum of these two states of water is collectively named freezable water $\left(W_{\mathrm{f}}\right)$ which exhibits a phase transition at a temperature of $0{ }^{\circ} \mathrm{C}$. On the other hand, the nonfreezable bound water $W_{\text {nfb }}$ interacts strongly with the polymer chains through hydrogen bonding and does not show any phase transition at $0{ }^{\circ} \mathrm{C}$. The sum of the freezable water $\left(W_{\mathrm{f}}\right)$ and non-freezable bound water $W_{\mathrm{nfb}}$ normalized by the weight of the hydrated hydrogel at equilibration $\left(M_{H G}\right)$ equals to the degree of hydration $\mathrm{DoH}$ as shown below in equation 8. Thus, the $\mathrm{DoH}$ determined by DSC may be directly compared with the $\mathrm{DoH}$ obtained by gravimetry and is generally expected to be in close agreement.

$D o H_{D S C}(\mathrm{wt} \%)=\frac{W_{\mathrm{f}}+W_{\mathrm{nfb}}}{M_{\mathrm{HG}}}=\frac{\left[W_{\mathrm{ff}}+W_{\mathrm{fb}}\right]+W_{\mathrm{nfb}}}{M_{\mathrm{HG}}}$

Figure 5 shows that warming the frozen hydrated hydrogels containing 3 mol\% TEGDA crosslinker with and without 5 mol\% DMAEMA resulted in a broad ice-melting endodermic peak at around $0^{\circ} \mathrm{C}$ correlated to the freezable free water in each hydrogel. The endodermic peak is smaller for hydrogels containing 5 mol\% DMAEMA suggesting less overall hydration. The broad peak at around $0^{\circ} \mathrm{C}$, representing freezable free water within the hydrogels, was split into two not so clearly resolved peaks which might be due to different ice structures of the freezable free water within the hydrogels [43]. Figure 5 also shows that the $0{ }^{\circ} \mathrm{C}$ peak areas of the hydrogels with DMAEMA were significantly lower than those of the hydrogels without DMAEMA. Besides the peak at around $0{ }^{\circ} \mathrm{C}$, the hydrogels 
without DMAEMA showed another relatively small broad peak at around $-6{ }^{\circ} \mathrm{C}$. This small broad peak could be due to the water that was loosely bound to the hydrogel polymer chains and could be frozen but at a temperature lower than $0{ }^{\circ} \mathrm{C}$ (depression of freezing point) [43-45]. Further observation showed that the small broad peak at around $-6^{\circ} \mathrm{C}$ was stronger in the hydrogels without DMAMEA that were equilibrated in HEPES buffer than the same hydrogel that was equilibrated in DI water, indicating that the buffer salts enhanced water to be bound to the polymer chains, consistent with the literature report [46]. As the areas of the endothermic peaks at around $0{ }^{\circ} \mathrm{C}$ and around $-6{ }^{\circ} \mathrm{C}$ correlate to the freezable free water content and freezable bound water content, respectively, we further measured the peak areas and calculated the freezable water content which equals the sum of freezable free water content and freezable bound water content. By using equation 6, we calculated the non-freezable bound water content from the $\mathrm{DoH}$ obtained from the gravimetric measurements at $25^{\circ} \mathrm{C}$ and the freezable water content obtained from the DSC results of the hydrogels and listed the results in Table 2.

Figure 5 here 


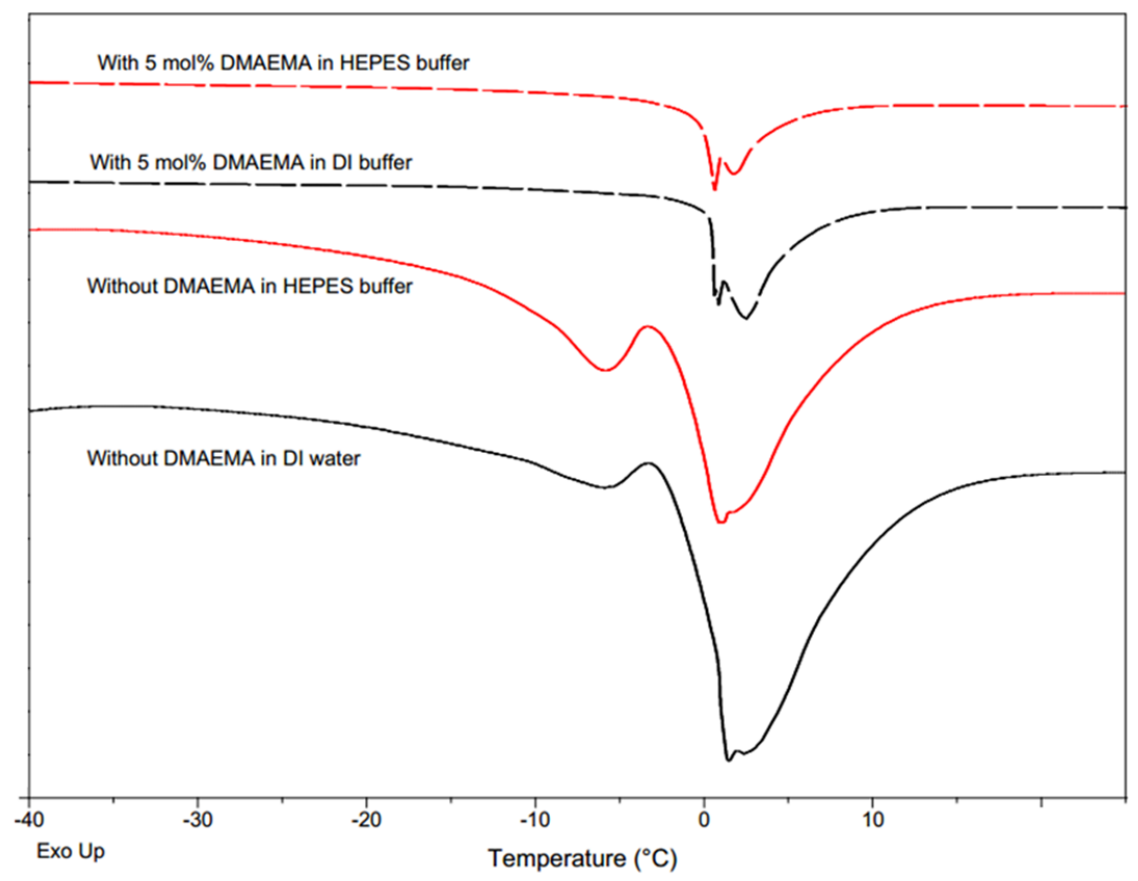

Figure 5. Endothermic curves of swollen hydrogels synthesized using 3 mol\% TEGDA with and without 5 mol\% DMAEMA.

Table 2. Distribution of equilibrium water content as determined by DSC for 3 mol\% TEGDA crosslinked hydrogel possessing 0 or 5 mol\% DMAEMA and equilibrated in DI water and HEPES buffer.

\begin{tabular}{|c|c|c|c|c|c|c|}
\hline \multirow[t]{2}{*}{ Hydrogels } & \multicolumn{2}{|c|}{$\begin{array}{l}\text { Degree of hydration at } \\
25^{\circ} \mathrm{C} \mathrm{DoH}(\mathrm{wt} \%)\end{array}$} & \multicolumn{2}{|c|}{$\begin{array}{l}\text { Freezable water } \\
\qquad \frac{W_{\mathrm{f}}}{M_{\mathrm{HG}}}(\mathrm{wt} \%)\end{array}$} & \multicolumn{2}{|c|}{$\begin{array}{l}\text { Non-Freezable bound water } \\
\qquad \frac{W_{\mathrm{nfb}}}{M_{\mathrm{HG}}}(\mathrm{wt} \%)\end{array}$} \\
\hline & $\mathrm{DI} \mathrm{H}_{2} \mathrm{O}$ & HEPES & $\mathrm{DI} \mathrm{H}_{2} \mathrm{O}$ & HEPES & $\mathrm{DI} \mathrm{H}_{2} \mathrm{O}$ & HEPES \\
\hline $\begin{array}{c}3 \text { mol\% TEGDA, } \\
0 \text { mol\% DMAEMA }\end{array}$ & $43.7 \pm 1.3$ & $43.4 \pm 0.5$ & $18.7 \pm 1.4$ & $18.6 \pm 0.3$ & $25.0 \pm 0.9$ & $24.8 \pm 0.6$ \\
\hline $\begin{array}{c}3 \text { mol\% TEGDA, } \\
5 \text { mol \% DMAEMA }\end{array}$ & $31.7 \pm 1.2$ & $29.7 \pm 1.1$ & $5.5 \pm 0.6$ & $3.8 \pm 0.5$ & $26.2 \pm 0.9$ & $25.9 \pm 0.9$ \\
\hline
\end{tabular}

Table 2 shows that a small amount $(5 \mathrm{~mol} \%)$ of the hydrophobic DMAEMA can dramatically alter the hydration characteristics of cross-linked poly(HEMA) by $24 \%$. From the results it can be 
observed that the major part of the equilibrium water content in the hydrogels with and without 5 mol\% DMAEMA is in non-freezable bound water state and addition of 5 mol\% DMAEMA did not have much influence on the non-freezable bound water amount. However, the addition of 5 mol\% DMAEMA significantly decreased the amount of freezable water three fold $(18.7 \pm 1.4 v s .5 .5 \pm 0.6$ in DI water; 18.6 $0.3 v$ s. $3.8 \pm 0.5$ in HEPES buffer) and thus the total hydration (43.7 $\pm 1.3 v s .31 .7 \pm 1.2$ in DI water; $43.4 \pm 0.5$ vs. $29.7 \pm 1.1$ buffer), and also disturbed the loose binding between the water and the polymer chains causing the disappearance of the small peak at around $-6^{\circ} \mathrm{C}$ in Figure 5. The reason might be because the non-freezable bound water is derived from water that is strongly bound to the polar hydroxyl groups of the poly(HEMA) repeat unit via hydrogen bonds [47] and since poly(HEMA) was the majority component with similar amount in both types of hydrogels one may expect the nonfreezable water content to be about the same. The freezable water is derived from the free or slightly bound water that resides within the nano-dimensioned pores of the hydrogels $[48,49]$. The addition of 5 mol\% DMAEMA could make the pores effectively smaller as the DMAEMA moiety $\left[\delta_{\text {DMAEMA }}=27.92\right.$ $\left.\left(\mathrm{cal} / \mathrm{cm}^{3}\right)^{1 / 2}\right]$ is more hydrophobic than the HEMA moiety $\left[\delta_{\mathrm{HEMA}}=31.2\left(\mathrm{cal} / \mathrm{cm}^{3}\right)^{1 / 2}\right][50-52]$, as reflected in their respective solubility parameters, $\delta$, calculated from group contributions. The presence of the HEPES buffer did not affect the non-freezable bound water content in both the hydrogels with and without $5 \mathrm{~mol} \%$ DMAEMA, and as well as the $\mathrm{DoH}$ and the freezable water contents in the hydrogels without DMAEMA. However, the presence of the HEPES buffer slightly decreased the $D o H$ and the freezable water contents in the hydrogels with 5 mol\% DMAEMA, probably due to the salting out effect as the DMAEMA is slightly positively charged at $\mathrm{pH}$ 7.4. The hydrogels without DMAEMA in both water and HEPES buffer had similar freezable water content but higher freezable bound water content in HEPES buffer (Figure 5), suggested that the salts in the buffer redistributed the freezable water within the hydrogels. Further investigation is needed to better understand the exact mechanisms for the observed redistribution of water content within the DMAEMA hydrogels, however, there is indication 
that this may arise from the unique meso-structure adopted by the amphiphilic nature of the hydrogel [53]. It is noteworthy that the $\mathrm{DoH}$ values at $25^{\circ} \mathrm{C}$ in Table 2 are similar to the corresponding gravimetrically determined $\mathrm{DoH}$ values at $37^{\circ} \mathrm{C}(43.7 \pm 1.3$ and $31.7 \pm 1.2 \%$ vs. $42 \pm 1.4$ and $34 \pm$ $1.2 \%$ in DI water; and $43.4 \pm 0.5$ and $29.7 \pm 1.1 \%$ vs. $41.3 \pm 0.8$ and $33.1 \pm 1.0$ in HEPES), suggesting that changing temperature from $37^{\circ} \mathrm{C}$ to $25^{\circ} \mathrm{C}$ did not appreciably affect the degree of hydration of the hydrogels.

\subsection{Glass transition temperature $\left(T_{\mathrm{g}}\right)$ of hydrogels}

DSC was used to determine the glass transition temperatures $\left(T_{\mathrm{g}}\right)$ of the hydrogels with and without 5 mol\% DMAEMA. The $T_{\mathrm{g}}$ values were obtained from the second heat cycle of the hydrogels dried through three different processes: vacuum $(23 \mathrm{inHg})$ at room temperature, vacuum $(23 \mathrm{inHg})$ at $80{ }^{\circ} \mathrm{C}$, and freeze drying are shown in Table 3 . The drying condition had appreciable influence on the measured $\mathrm{Tg}$. For the hydrogels without $5 \mathrm{~mol} \%$ DMAEMA, vacuum drying at $80{ }^{\circ} \mathrm{C}$ caused the $T_{\mathrm{g}}$ to shift to higher temperature than the vacuum drying at room temperature, and the freeze drying shifted the $T_{\mathrm{g}}$ to an even higher temperature than the vacuum drying at $80{ }^{\circ} \mathrm{C}$. For the hydrogels with $5 \mathrm{~mol} \%$ DMAEMA, the vacuum drying at room temperature and the freeze drying produced a similar $T_{\mathrm{g}}$, while the vacuum drying at $80{ }^{\circ} \mathrm{C}$ caused the $T_{\mathrm{g}}$ to shift to higher temperature than the other two drying processes. The reason might be that the different drying processes resulted in the hydrogels having different amounts of residual water which acted as a plasticizer to decrease the $T_{\mathrm{g}}$. The vacuum drying at $80{ }^{\circ} \mathrm{C}$ and freeze drying made the hydrogels drier (containing less water) than the vacuum drying at room temperature. Depending on the chemical structures of the hydrogels (with or without DMAEMA), the vacuum drying at $80{ }^{\circ} \mathrm{C}$ could cause the hydrogels to contain more or less water than freeze drying. More importantly, addition of 5 mol\% of DMAEMA, which reduced overall hydration by reducing free water (above), caused the $T_{\mathrm{g}}$ to shift to a higher temperature under all the three drying processes. The 
reason might be due to the hydrophobicity of the DMAE moiety and clearly correlates with the water distribution discussed above.

Table 3. The glass transition temperatures $\left(T_{\mathrm{g}}\right)$ from the second heat cycle for the hydrogels with and without 5 mol\% DMAEMA under different drying processes.

\begin{tabular}{|c|c|c|c|c|c|c|}
\hline \multirow{2}{*}{$\overbrace{\text { Hydrogel }}^{T_{\mathrm{g}}}$} & \multicolumn{2}{|c|}{ Vacuum dry at room temperature } & \multicolumn{2}{|c|}{ Vacuum dry at $80^{\circ} \mathrm{C}$} & \multicolumn{2}{|c|}{ Freeze dry } \\
\hline & Onset & Midpoint & Onset & Midpoint & Onset & Midpoint \\
\hline AAEM & 47 & 67 & 69.6 & 75 & 80.0 & 89.7 \\
\hline With DMAEMA & 79.5 & 100.0 & 102.8 & 111.1 & 85.9 & 98.2 \\
\hline
\end{tabular}

\subsection{Influence of concentration of CBB and crosslink density of hydrogels}

Figure 6 reveals the influence of cross-link density on the partition coefficient, $\Phi$, as a function of mol\% TEGDA at a fixed CBB concentration as determined in a CBB/HEPES solution of $6.0 \mathrm{wt} \% \mathrm{CBB}$ at $37{ }^{\circ} \mathrm{C}$. Figure 6 is typical of the dependence of the partition coefficient, $\Phi$, on cross-linker concentration. The partition coefficient increases linearly with the increase in cross-linker concentration used in the fabrication of the hydrogel. The average partition coefficient was found to be $6.69 \pm 0.12$ (Table 4) with systematic but small increases among hydrogel compositions of varying TEGDA

Table 4. Crosslink density dependence on the average of the CBB partition coefficient, $\Phi$, as determined for each mol\% TEGDA but derived from a range of evaluated starting concentrations $1-6 \mathrm{wt} \%$ of $\mathrm{CBB}$ at $37^{\circ} \mathrm{C}$.

\begin{tabular}{|l|l|l|l|l|l|l|l|}
\hline $\begin{array}{l}\text { Mol } \\
\text { TEGDA }\end{array}$ & $1 \mathrm{~mol} \%$ & $3 \mathrm{~mol} \%$ & $5 \mathrm{~mol} \%$ & $7 \mathrm{~mol} \%$ & $9 \mathrm{~mol} \%$ & $12 \mathrm{~mol} \%$ & Average \\
\hline $\begin{array}{l}\text { Partition } \\
\text { Coefficient, (Ф) }\end{array}$ & 6.54 & 6.62 & 6.72 & 6.81 & 6.59 & 6.84 & $6.69 \pm 0.12$ \\
\hline
\end{tabular}




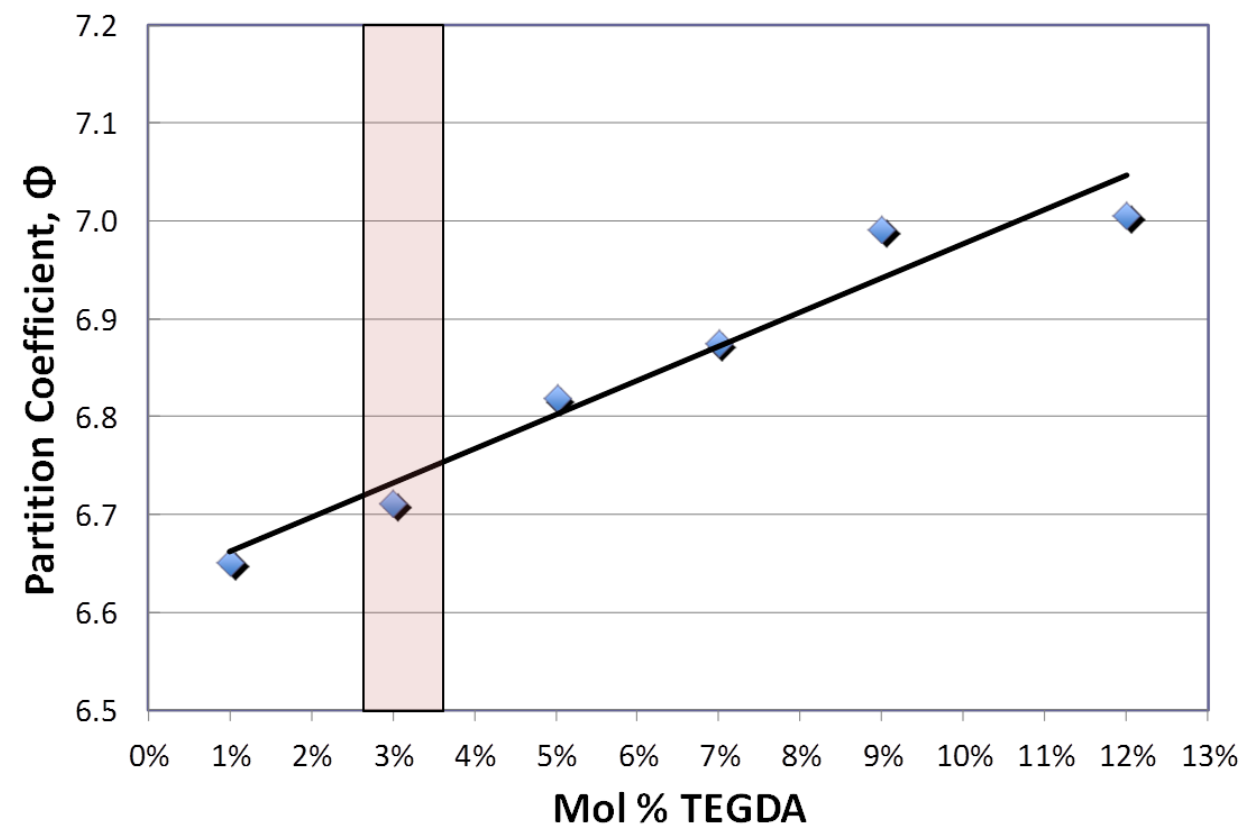

Figure 6. Partition coefficient, $\Phi$, of $\mathrm{CBB}$ for non-DMAEMA containing hydrogels of varying crosslink density (1-12 mol\% TEGDA) as determined in a CBB/HEPES solution of exclusively $6.0 \mathrm{wt} \% \mathrm{CBB}$ at $37^{\circ} \mathrm{C}$.

The CBB concentration dependence of the sorption capacity $\mathrm{K}$ into poly(HEMA)-based hydrogels was studied as a function of crosslink density over the range of 1-12 mol\% TEGDA and is shown in Figure 7. The equilibrium partitioning of CBB into hydrogels shows clear concentration dependence. It rises sharply at low concentrations and plateaus at ca. 1 wt $\%$ concentration and was found to reveal the same profile regardless of the crosslink density. Moreover, the absorption profile resembles the classic adsorption isotherm [54]. The Langmuir and Freundlich adsorption isotherm models were explored for their possibility to describe the experimental isotherm data within the hydrogels [55]. These models are represented by the following equations, respectively (H.M.F. Freundlich, 1906; Langmuir, 1918):

$$
\mathrm{K}=\frac{\mathrm{K}_{\max } \mathrm{BC}_{\mathrm{CB}}}{1+\mathrm{BC}_{\mathrm{CB}}}
$$




$$
\mathrm{K}=\mathrm{K}_{\mathrm{F}} \mathrm{C}_{\mathrm{CB}} 1 / \mathrm{n}
$$

where $\mathrm{K}$ is the sorption capacity reflecting the amount of $\mathrm{CBB}$ absorbed/adsorbed per gram of hydrogel at equilibrium $\left(\mathrm{mg} \cdot \mathrm{g}^{-1}\right), \mathrm{K}_{\max }$ is the maximum sorption capacity reflecting the maximal absorption/adsorption capacity of the hydrogel $\left(\mathrm{mg} \cdot \mathrm{g}^{-1}\right)$, and $C_{C B}$ is the equilibrium concentration of the CBB solution $\left(\mathrm{mg} \cdot \mathrm{L}^{-1}\right)$. In the Langmuir model (equation 9), $B$ is related to the energy of adsorption $\left(\mathrm{L} \cdot \mathrm{mg}^{-1}\right)$, whereas $K_{F}$ and $n$ (equation 10) are the Freundlich adsorption constants, indicative of the relative capacity and the adsorption intensity, respectively [56]. Plots of $1 / \mathrm{K} v s$. $1 / C_{C B}$, reflecting the linearized form of the Langmuir equation resulted in an average $R^{2}$ that was $0.996 \pm 0.001$ while plots $\ln (\mathrm{K})$ vs. $\ln \left(\mathrm{C}_{\mathrm{CB}}\right)$, reflecting the linearized form of the Freundlich equation resulted in an average $\mathrm{R}^{2}$ that was $0.834 \pm 0.016$. This confirmed the suitability of the Langmuir model for describing the partitioning of CBB into the poly(HEMA)-based hydrogels. Parameter estimates extracted from the linear forms of the above equations were used along with the solver add-in capability within Microsoft Excel to iteratively converge upon best fit values for the non-linear form of the equation. The $\mathrm{K}_{\max }$ was seen to increase and the $B$ parameter to decrease monotonically with increasing TEGDA cross-linker concentration. $\mathrm{K}_{\max }$ increased from 6.80 (3 mol\%) to $7.17(12 \mathrm{~mol} \%)$ as a function of TEGDA mol\% or cross-link density in accord with a monotonic reduction of the degree of hydration (above). The average $\mathrm{K}_{\max }$ calculated in this way was $6.99 \pm 0.15$. Thus the sorption capacity increases and the energy term decreases with increasing network cross-link density.

If the CBB partitioned into the free water held within the nano-voids of the hydrogel, then $\mathrm{K}_{\max }$ will be expected to increase with an increase in hydration. Quite the opposite occurs. If on the other hand the $\mathrm{CBB}$ partitioned by adsorption onto effective surfaces that subtend the interface between the 
fluid in the nano-void and the hydrogel chemistry then this may rationalize; 1) the adherence to the Langmuir model and 2) the increase in capacity with an increase in cross-linker density.

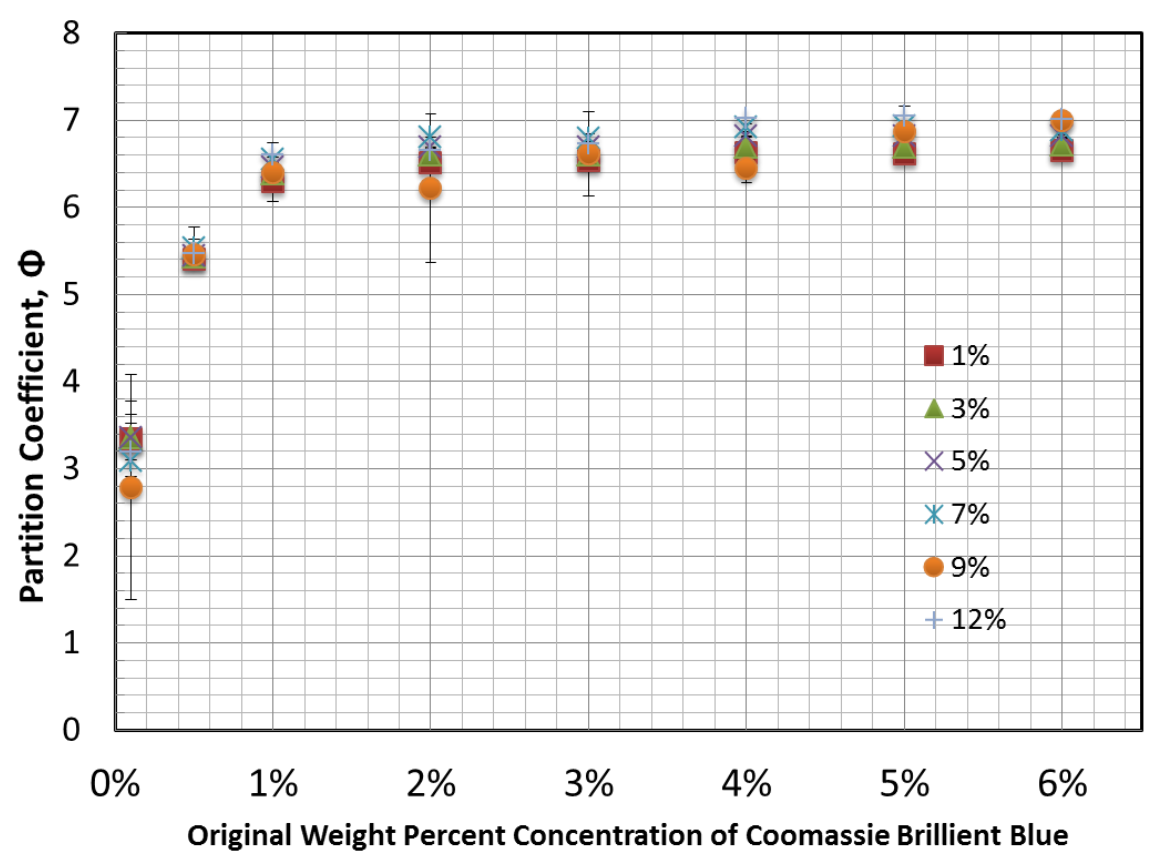

Figure 7. Partition coefficient of CBB for non-DMAEMA containing hydrogels of varying crosslink density (1-12 mol\% TEGDA) as determined in CBB/HEPES solutions of varying initial concentrations $(0.1-6.0 \mathrm{wt} \% \mathrm{CBB})$ at $37^{\circ} \mathrm{C}$.

\subsection{Influence of molecular composition of the hydrogel}

Freshly prepared and UV-crosslinked hydrogel discs all began at a volume of $15.9 \mathrm{~mm}^{3}$. Hydrogels containing DMAEMA swelled to $45.8 \mathrm{~mm}^{3}$. Exclusion of DMAEMA at 3 mol\% TEGDA crosslink density resulted in a larger hydrogel volume of $87.1 \mathrm{~mm}^{3}$. The presence of a $5 \mathrm{~mol} \%$ DMAEMA in a 3 mol\% TEGDA cross-linked hydrogel resulted in a decrease in swollen hydrogel volume by $90 \%$. Significantly less CBB partitioned into hydrogels lacking DMAEMA (Figure 8). No appreciable changes to CBB loading were observed accompanying shifts in $\mathrm{pH}$ that crosses the known pKa of DMAEMA (7.5). Exclusion of DMAEMA from the formula of hydrogels significantly increased 
hydrogel swelling and reduced the partition coefficient of the hydrogels for CBB. Controlled adsorption of cationic dyes, Toluidine Blue for example, into hydrogels has been achieved through incorporation of negatively charged functional groups such as maleic acid $[57,58]$. The decrease in CBB loading with exclusion of DMAEMA is to be expected as DMAEMA $\left(\mathrm{pK}_{\mathrm{a}}=7.3-7.5\right)$ has an associated positive charge while $\mathrm{CBB}$ has an associated negative charge at physiologic $\mathrm{pH}$ [59-61]. However, this cannot account for all of the observed difference since the DMAEMA is only at $5 \mathrm{~mol} \%$ and there is no apparent $\mathrm{pH}$ dependence of the partition coefficient. The $\mathrm{pH}$ independence is likely due to the hydrogel's internal $\mathrm{pH}$ stability. The hydrogels used in this body of work were originally designed to protect the activity of biomolecules such as glucose and lactate oxidase enzymes from changes in $\mathrm{pH}$ $[62,63]$ and have also be the basis for $\mathrm{pH}$ responsive drug delivery e.g.insulin release [15, 17]. A simple calculation of the mole ratio shows that at 5 mol \% DMAEMA there are 1.7 molecules of DMAEMA per molecule of CBB partitioned.

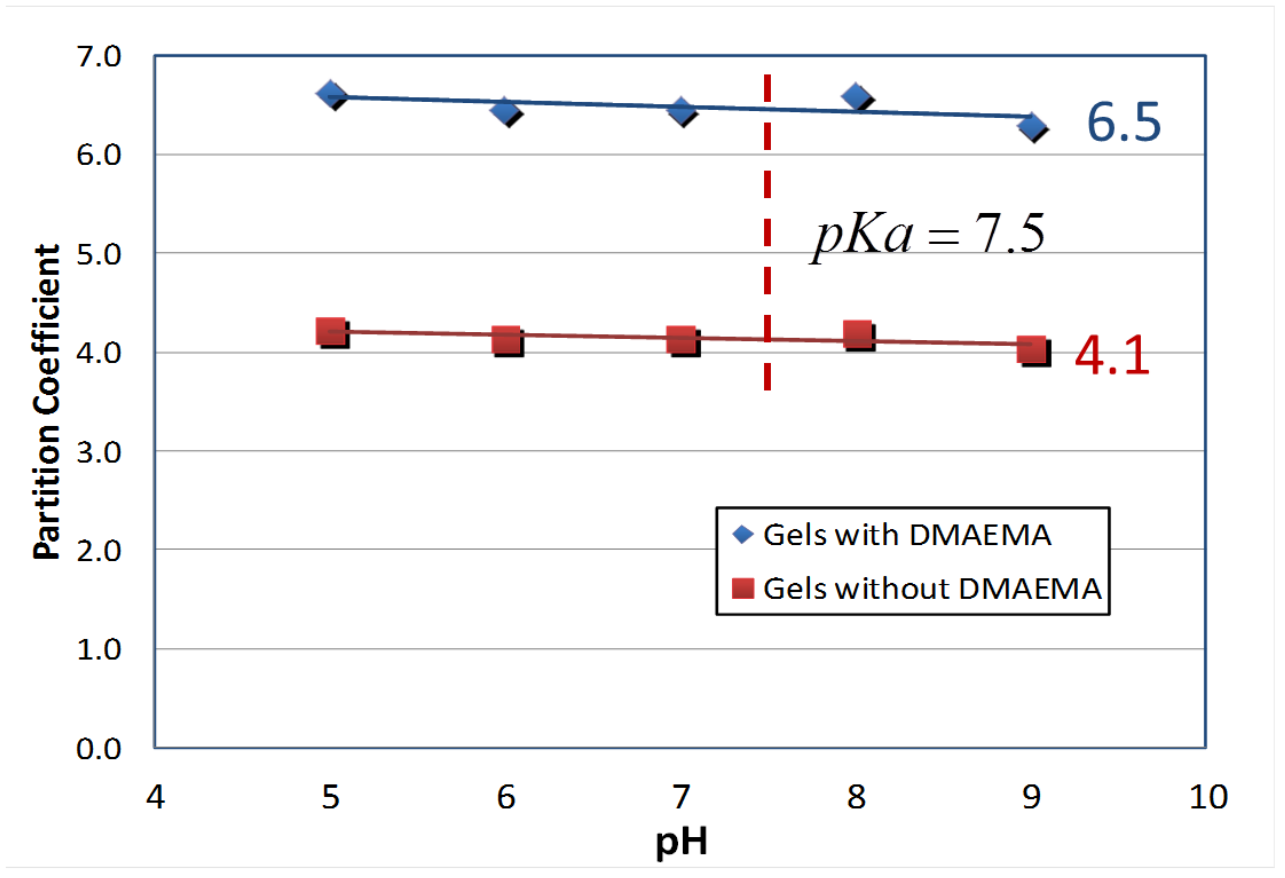

Figure 8. Partition coefficient of CBB for a hydrogel of $3 \mathrm{~mol} \%$ TEGDA with or without $5 \mathrm{~mol} \%$ DMAEMA obtained in a CBB/HEPES solution of $6.0 \mathrm{wt} \% \mathrm{CBB}$ at $37^{\circ} \mathrm{C}$. 
Crosslink density is known to affect tortuosity, degree of hydration and volume of hydrogels. Incorporation of DMAEMA alone is sufficient to increase partitioning regardless of the crosslink density or swelling of the hydrogel. However, the high degree of partitioning in the hydrogels lacking in DMAEMA is indicative of additional mechanisms for partitioning. Reduced swelling of gels containing DMAEMA can be due to DMAEMA serving as a physical cross-linking agent $[64,65]$ within the hydrogel via hydrogen bonding. These strong hydrogen bonds can prevent some association of the amino groups with water [66] resulting in a tight network and reduced $\mathrm{pH}$ dependence of swelling. At 5 mol\% DMAEMA the hydrogen bonding may be dominating the hydrogel swelling characteristics over the chemical crosslink density. Additional tests examining hydrogel swelling and CBB loading as a function of DMAEMA concentration at a fixed, low concentration of TEGDA will be pursued in the future.

\section{CONCLUSIONS}

The poly(HEMA)-based hydrogels demonstrated a decrease in hydration (46\% to $31 \%$ ) with an increase in cross-linker concentration or cross-link density (1-12 mol\% TEGDA). The incorporation of 5 mol\% DMAEMA into HEMA based hydrogels reduced the overall hydration by deionized water (35\% to $33 \%$ ) and altered the distribution of water types among freezable, freezable-bound water (freezing point depressed) and non-freezable water with the net effect being a reduction of hydration at the expense of freezable water content (from 18.7\% - 5.5\%). Freeze drying produced the most consistent drying condition. The CBB loading capacity of hydrogels was subtly increased by the influence of crosslinker concentration; with an increase in capacity accompanying an increase in cross-link density (6.54 6.84 at $6 \mathrm{wt} \% \mathrm{CBB})$. This increase in cross-link density was accompanied by a reduction in overall hydration and increase in partitioning capacity and so hydration was not the principal driver for CBB partitioning into these hydrogels. The partition coefficient followed a Langmuir adsorption isotherm 
model with a maximal capacity that increased with cross-link density [from 6.80 (3 mol \%) to 7.17 (12 mol \%)]. This implied that partitioning of $\mathrm{CBB}$ into poly(HEMA)-based hydrogels was akin to adsorption onto a highly porous solid (e.g. activated carbon), in which the surface site density may be engineered by the cross-link density.

\section{ACKNOWLEDGEMENTS}

The authors acknowledge support from the US Department of Defense (DoDPRMRP) grant PR023081/DAMD17-03-1-0172 and the Consortium of the Clemson University Center for Bioelectronics, Biosensors and Biochips (C3B). A.M. Wilson acknowledges support from the Department of Chemistry, University of the West Indies, St. Augustine and ABTECH Scientific, Inc. 


\subsection{REFERENCES}

\section{References}

[1] Paradiso P, Galante R, Santos L, Alves de Matos A, Colaço R, Serro A, et al. Comparison of two hydrogel formulations for drug release in ophthalmic lenses. Journal of Biomedical Materials Research Part B: Applied Biomaterials. 2014.

[2] Radhakrishnan J, Krishnan UM, Sethuraman S. Hydrogel based injectable scaffolds for cardiac tissue regeneration. Biotechnology advances. 2014.

[3] Rezaei S, Ishak Z. Grafting of collagen onto interpenetrating polymer networks of poly (2hydroxyethyl methacrylate) and poly (dimethyl siloxane) polymer films for biomedical applications. Express Polymer Letters. 2014;8.

[4] Yom-Tov O, Frisman I, Seliktar D, Bianco-Peled H. A novel method for hydrogel nanostructuring. European Polymer Journal. 2014.

[5] Farhat S, Gilliam M, Rabago-Smith M, Baran C, Walter N, Zand A. Polymer coatings for biomedical applications using atmospheric pressure plasma. Surface and Coatings Technology. 2014;241:123-9.

[6] Sastry T. Silk Fibroin Coated with Poly 2-hydroxyethyl Methacrylate Impregnated with Silver Nanoparticles Coupled with Ciprofloxacin as a Biomaterial for Biomedical Applications. Trends in Biomaterials and Artificial Organs. 2014;28.

[7] Kotanen CN, Guiseppi-Elie A. Bioactive electroconductive hydrogels yield novel biotransducers for glucose. Macromolecular Symposia: Wiley Online Library; 2012. p. 187-97.

[8] Le Droumaguet B, Lacombe R, Ly H-B, Guerrouache M, Carbonnier B, Grande D. Engineering functional doubly porous PHEMA-based materials. Polymer. 2014;55:373-9.

[9] Bhowmick A, Saha A, Pramanik N, Banerjee S, Das M, Kundu PP. Novel magnetic antimicrobial nanocomposites for bone tissue engineering applications. RSC Advances. 2015;5:25437-45.

[10] Chen B, Chen J, Yang L, Zhao G, Ding G. Electrodepositing salicylate modified PHEMA on stainless steel surface for hemocompatibility. Surface Engineering. 2014;30:768-75.

[11] Poinern GEJ, Ali N, Le X, Fawcett D. Nano-hardness and elastic modulus of anodic aluminium oxide based Poly (2-hydroxyethylmethacrylate) composite membranes. AIMS Materials Science. 2014;1:159-73.

[12] García-Millán E, Koprivnik S, Otero-Espinar FJ. Drug loading optimization and extended drug delivery of corticoids from pHEMA based soft contact lenses hydrogels via chemical and microstructural modifications. International journal of pharmaceutics. 2015;487:260-9.

[13] Wilson AN, Guiseppi-Elie A. Bioresponsive hydrogels. Adv Healthc Mater. 2013;2:520-32.

[14] Wilson AN, Guiseppi-Elie A. Targeting homeostasis in drug delivery using bioresponsive hydrogel microforms. International journal of pharmaceutics. 2014;461:214-22.

[15] Guiseppi-Elie A, Brahim SI, Narinesingh D. A Chemically Synthesized Artificial Pancreas: Release of Insulin from Glucose-Responsive Hydrogels. Advanced Materials. 2002;14:743-6. 
[16] Zan M, Li J, Luo S, Ge Z. Dual pH-triggered multistage drug delivery systems based on host-guest interaction-associated polymeric nanogels. Chem Commun. 2014;50:7824-7.

[17] Brahim S, Narinesingh D, Guiseppi-Elie A. Release characteristics of novel pH-sensitive $p$ (HEMA-DMAEMA) hydrogels containing 3-(trimethoxy-silyl) propyl methacrylate. Biomacromolecules. 2003;4:1224-31.

[18] Guiseppi-Elie A. Electroconductive hydrogels: synthesis, characterization and biomedical applications. Biomaterials. 2010;31:2701-16.

[19] Abraham S, Brahim S, Ishihara K, Guiseppi-Elie A. Molecularly engineered p (HEMA)-based hydrogels for implant biochip biocompatibility. Biomaterials. 2005;26:4767-78.

[20] Guiseppi-Elie A, Dong C, Dinu CZ. Crosslink density of a biomimetic poly (HEMA)-based hydrogel influences growth and proliferation of attachment dependent RMS 13 cells. Journal of Materials Chemistry. 2012;22:19529-39.

[21] Kotanen CN, Wilson AN, Wilson AM, Ishihara K, Guiseppi-Elie A. Biomimetic hydrogels gate transport of calcium ions across cell culture inserts. Biomedical microdevices. 2012;14:549-58.

[22] Lewis CL, Anthamatten M. Synthesis, swelling behavior, and viscoelastic properties of functional poly(hydroxyethyl methacrylate) with ureidopyrimidinone side-groups. Soft Matter. 2013;9:4058-66.

[23] Morita S. Hydrogen-bonds structure in poly(2-hydroxyethyl methacrylate) studied by temperaturedependent infrared spectroscopy. Frontiers in Chemistry. 2014;2:10.

[24] Lord MS, Stenzel MH, Simmons A, Milthorpe BK. The effect of charged groups on protein interactions with poly(HEMA) hydrogels. Biomaterials. 2006;27:567-75.

[25] Wilson A, Blenner M, Guiseppi-Elie A. Polyplex Formation Influences Release Mechanism of Mono- and Di-Valent Ions from Phosphorylcholine Group Bearing Hydrogels. Polymers. 2014;6:245172.

[26] van de Wetering P, Zuidam NJ, van Steenbergen MJ, van der Houwen OAGJ, Underberg WJM, Hennink WE. A Mechanistic Study of the Hydrolytic Stability of Poly(2-(dimethylamino)ethyl methacrylate). Macromolecules. 1998;31:8063-8.

[27] Wilson AN, Guiseppi-Elie A. Targeting homeostasis in drug delivery using bioresponsive hydrogel microforms. International Journal of Pharmaceutics. 2014;461:214-22.

[28] Gordijo CR, Koulajian K, Shuhendler AJ, Bonifacio LD, Huang HY, Chiang S, et al. Nanotechnology-Enabled Closed Loop Insulin Delivery Device: In Vitro and In Vivo Evaluation of Glucose-Regulated Insulin Release for Diabetes Control. Advanced Functional Materials. 2011;21:7382.

[29] Goldenberg M, Beekman A. Detection of protein deposition on contact lens type polymeric hydrogels by Coomassie blue R staining. Biomaterials. 1991;12:267-74.

[30] Pan G, Guo B, Ma Y, Cui W, He F, Li B, et al. Dynamic Introduction of Cell Adhesive Factor via Reversible Multicovalent Phenylboronic Acid/cis-Diol Polymeric Complexes. Journal of the American Chemical Society. 2014;136:6203-6.

[31] Bayramoğlu G, Yılmaz M, Batislam E, Arıca MY. Heparin-coated poly (hydroxyethyl methacrylate/albumin) hydrogel networks: In vitro hemocompatibility evaluation for vascular biomaterials. Journal of applied polymer science. 2008;109:749-57. 
[32] Liu J, Liu H, Kang H, Donovan M, Zhu Z, Tan W. Aptamer-incorporated hydrogels for visual detection, controlled drug release, and targeted cancer therapy. Analytical and bioanalytical chemistry. 2012;402:187-94.

[33] Perçin I, Sağlar E, Yavuz H, Aksöz E, Denizli A. Poly (hydroxyethyl methacrylate) based affinity cryogel for plasmid DNA purification. International journal of biological macromolecules. 2011;48:57782.

[34] Lai R, Liu H, Lee WH, Zhang Y. An anionic antimicrobial peptide from toad Bombina maxima. Biochemical and biophysical research communications. 2002;295:796-9.

[35] Schittek B, Hipfel R, Sauer B, Bauer J, Kalbacher H, Stevanovic S, et al. Dermcidin: a novel human antibiotic peptide secreted by sweat glands. Nature immunology. 2001;2:1133-7.

[36] Hoare TR, Kohane DS. Hydrogels in drug delivery: progress and challenges. Polymer. 2008;49:1993-2007.

[37] Wang L-S, Boulaire J, Chan PP, Chung JE, Kurisawa M. The role of stiffness of gelatinhydroxyphenylpropionic acid hydrogels formed by enzyme-mediated crosslinking on the differentiation of human mesenchymal stem cell. Biomaterials. 2010;31:8608-16.

[38] Lee KY, Bouhadir KH, Mooney DJ. Degradation behavior of covalently cross-linked poly (aldehyde guluronate) hydrogels. Macromolecules. 2000;33:97-101.

[39] Pergushov DV, Muller AHE, Schacher FH. Micellar interpolyelectrolyte complexes. Chemical Society Reviews. 2012;41:6888-901.

[40] Kabanov VA, Skobeleva VB, Rogacheva VB, Zezin AB. Sorption of Proteins by Slightly CrossLinked Polyelectrolyte Hydrogels: Kinetics and Mechanism. The Journal of Physical Chemistry B. 2004;108:1485-90.

[41] Boztas AO, Guiseppi-Elie A. Immobilization and release of the redox mediator ferrocene monocarboxylic acid from within cross-linked p (HEMA-co-PEGMA-co-HMMA) hydrogels. Biomacromolecules. 2009;10:2135-43.

[42] Peppas NA. Kinetics of Smart Hydrogels. In: Yui N, Mrsny RJ, Park K, editors. Reflexive Polymers and Hydrogels: Understanding and Designing Fast Responsive Polymeric Systems. New York: CRC Press Taylor and Francis Division; 2004. p. 472.

[43] Li W, Xue F, Cheng R. States of water in partially swollen poly (vinyl alcohol) hydrogels. Polymer. 2005;46:12026-31.

[44] Ostrowska-Czubenko J, Pieróg M, Gierszewska-Drużyńska M. State of water in noncrosslinked and crosslinked hydrogel chitosan membranes-DSC studies. Progr Chem Appl Chitin Deriv. 2011;16:14756.

[45] Ping ZH, Nguyen QT, Chen SM, Zhou JQ, Ding YD. States of water in different hydrophilic polymers — DSC and FTIR studies. Polymer. 2001;42:8461-7.

[46] Yudianti R, Karina M, Sakamoto M, Azuma J-i. DSC analysis on water state of salvia hydrogels. Macromolecular Research. 2009;17:1015-20.

[47] Tanaka M, Mochizuki A. Effect of water structure on blood compatibility - thermal analysis of water in poly(meth)acrylate. Journal of Biomedical Materials Research Part A. 2004;68A:684-95. 
[48] Topgaard D, Söderman O. Diffusion of Water Absorbed in Cellulose Fibers Studied with 1H-NMR. Langmuir. 2001;17:2694-702.

[49] Topgaard D, Söderman O. Self-Diffusion of Nonfreezing Water in Porous Carbohydrate Polymer Systems Studied with Nuclear Magnetic Resonance. Biophysical Journal. 2002;83:3596-606.

[50] Çaykara T, Özyürek C, Kantoğlu Ö, Güven O. Influence of gel composition on the solubility parameter of poly(2-hydroxyethyl methacrylate-itaconic acid) hydrogels. Journal of Polymer Science Part B: Polymer Physics. 2002;40:1995-2003.

[51] Kwok AY, Qiao GG, Solomon DH. Synthetic hydrogels 3. Solvent effects on poly(2-hydroxyethyl methacrylate) networks. Polymer. 2004;45:4017-27.

[52] Mandal S, Pangarkar VG. Pervaporative dehydration of 1-methoxy propanol with acrylonitrile based co-polymer membranes prepared through emulsion polymerization: a solubility parameter approach and study of structural impact. Journal of Membrane Science. 2002;209:53-66.

[53] Shang K, Shan G, Pan P. Unique multiple soluble-insoluble phase transitions in aqueous two-phase copolymerization of acrylamide and a weakly charged comonomer. Soft Matter. 2014;10:8913-22.

[54] Giles CH, Smith D, Huitson A. A general treatment and classification of the solute adsorption isotherm. I. Theoretical. Journal of Colloid and Interface Science. 1974;47:755-65.

[55] Soleri R, Demey H, Tria SA, Guiseppi-Elie A, Ibn Had Hassine A, Gonzalez C, et al. Peptide conjugated chitosan foam as a novel approach for capture-purification and rapid detection of hapten Example of ochratoxin A. Biosens Bioelectron. 2015;67:634-41.

[56] Ho Y-S. Isotherms for the Sorption of Lead onto Peat: Comparison of Linear and Non-Linear Methods. Polish Journal of Environmental Studies. 2006;15:81-6.

[57] Saraydin D, Karadăg E, Güven O. Adsorption of Some Basic Dyes by Acrylamide-Maleic Acid Hydrogels. Separation Science and Technology. 1996;31:423-34.

[58] KaradaĞ E, TopaÇ F, Kundakci S, ÜZÜM Ö. Novel composite sorbent AAm/MA hydrogels containing starch and kaolin for water sorption and dye uptake. Bulletin of Materials Science. 2014;37:1637-46.

[59] Fournier D, Hoogenboom R, Thijs HM, Paulus RM, Schubert US. Tunable pH-and temperaturesensitive copolymer libraries by reversible addition-fragmentation chain transfer copolymerizations of methacrylates. Macromolecules. 2007;40:915-20.

[60] Nouri A, Castro R, Kairys V, Santos JL, Rodrigues J, Li Y, et al. Insight into the role of N, Ndimethylaminoethyl methacrylate (DMAEMA) conjugation onto poly (ethylenimine): cell viability and gene transfection studies. Journal of Materials Science: Materials in Medicine. 2012;23:2967-80.

[61] Chial H, Thompson H, Splittgerber A. A spectral study of the charge forms of Coomassie blue G. Analytical biochemistry. 1993;209:258-66.

[62] Brahim S, Narinesingh D, Guiseppi-Elie A. Bio-smart materials: Kinetics of immobilized enzymes in $\mathrm{p}(\mathrm{HEMA}) / \mathrm{p}$ (pyrrole) hydrogels in amperometric biosensors. Macromolecular Symposia. 2002; 186:63-73.

[63] Brahim S, Narinesingh D, Guiseppi-Elie A. Bio-smart hydrogels: Co-joined molecular recognition and signal transduction in biosensor fabrication and drug delivery. Biosensors and Bioelectronics. 2002; 17:973-81. 
[64] Hennink W, Van Nostrum C. Novel crosslinking methods to design hydrogels. Advanced drug delivery reviews. 2012;64:223-36.

[65] Song G, Zhang L, He C, Fang D-C, Whitten PG, Wang H. Facile Fabrication of Tough Hydrogels Physically Cross-Linked by Strong Cooperative Hydrogen Bonding. Macromolecules. 2013;46:7423-35.

[66] Cho SH, Jhon MS, Yuk SH, Lee HB. Temperature-induced phase transition of poly (N, Ndimethylaminoethyl methacrylate-co-acrylamide). Journal of Polymer Science Part B: Polymer Physics. 1997;35:595-8. 


\section{List of Tables}

Table 1. Monomer cocktail formulations for the preparation of varying mol\% TEGDA crosslinked poly(HEMA)-based hydrogels containing DMAEMA (left) and no DMAEMA (right) prepared in triplicate.

\begin{tabular}{|l|c|c|c|c|c|c|c|c|c|c|c|c|}
\hline $\begin{array}{l}\text { Crosslink density } \\
\text { (mol\%) }\end{array}$ & \multicolumn{2}{|c|}{1} & \multicolumn{2}{|c|}{3} & \multicolumn{2}{c|}{5} & \multicolumn{2}{c|}{7} & \multicolumn{3}{c|}{12} \\
\hline HEMA & 81 & 86 & 79 & 84 & 77 & 82 & 75 & 80 & 73 & 78 & 70 & 75 \\
\hline TEGDA & 1 & 1 & 3 & 3 & 5 & 5 & 7 & 7 & 9 & 9 & 12 & 12 \\
\hline OEG(400)MA (n=10) & 5 & 5 & 5 & 5 & 5 & 5 & 5 & 5 & 5 & 5 & 5 & 5 \\
\hline HMMA & 5 & 5 & 5 & 5 & 5 & 5 & 5 & 5 & 5 & 5 & 5 & 5 \\
\hline PNVP & 2 & 2 & 2 & 2 & 2 & 2 & 2 & 2 & 2 & 2 & 2 & 2 \\
\hline DMAEMA & 5 & 0 & 5 & 0 & 5 & 0 & 5 & 0 & 5 & 0 & 5 & 0 \\
\hline DMPA & 1 & 1 & 1 & 1 & 1 & 1 & 1 & 1 & 1 & 1 & 1 & 1 \\
\hline
\end{tabular}

Table 2. Distribution of equilibrium water content as determined by DSC for 3 mol\% TEGDA crosslinked hydrogel possessing 0 or 5 mol\% DMAEMA and equilibrated in DI water and HEPES buffer.

\begin{tabular}{|c|c|c|c|c|c|c|}
\hline \multirow[t]{2}{*}{ Hydrogels } & \multicolumn{2}{|c|}{$\begin{array}{c}\text { Degree of hydration at } \\
25^{\circ} \mathrm{C} \mathrm{DoH}(\mathrm{wt} \%)\end{array}$} & \multicolumn{2}{|c|}{$\begin{array}{l}\text { Freezable water } \\
\qquad \frac{W_{\mathrm{f}}}{M_{\mathrm{HG}}}(\mathrm{wt} \%)\end{array}$} & \multicolumn{2}{|c|}{$\begin{array}{l}\text { Non-Freezable bound water } \\
\qquad \frac{W_{\mathrm{nfb}}}{M_{\mathrm{HG}}}(\mathrm{wt} \%)\end{array}$} \\
\hline & $\mathrm{DI} \mathrm{H}{ }_{2} \mathrm{O}$ & HEPES & $\mathrm{DI} \mathrm{H}{ }_{2} \mathrm{O}$ & HEPES & $\mathrm{DI} \mathrm{H}_{2} \mathrm{O}$ & HEPES \\
\hline $\begin{array}{c}3 \text { mol\% TEGDA, } \\
0 \text { mol\% DMAEMA }\end{array}$ & $43.7 \pm 1.3$ & $43.4 \pm 0.5$ & $18.7 \pm 1.4$ & $18.6 \pm 0.3$ & $25.0 \pm 0.9$ & $24.8 \pm 0.6$ \\
\hline $\begin{array}{c}3 \text { mol\% TEGDA, } \\
5 \text { mol \% DMAEMA }\end{array}$ & $31.7 \pm 1.2$ & $29.7 \pm 1.1$ & $5.5 \pm 0.6$ & $3.8 \pm 0.5$ & $26.2 \pm 0.9$ & $25.9 \pm 0.9$ \\
\hline
\end{tabular}


Table 3. The glass transition temperatures $\left(T_{\mathrm{g}}\right)$ from the second heat cycle for the hydrogels with and without 5 mol\% DMAEMA under different drying processes.

\begin{tabular}{|c|c|c|c|c|c|c|}
\hline \multirow{2}{*}{$\overbrace{\text { Hydrogel }}^{T_{\mathrm{g}}}$} & \multicolumn{2}{|c|}{ Vacuum dry at room temperature } & \multicolumn{2}{|c|}{ Vacuum dry at $80^{\circ} \mathrm{C}$} & \multicolumn{2}{|c|}{ Freeze dry } \\
\hline & Onset & Midpoint & Onset & Midpoint & Onset & Midpoint \\
\hline MAEMA & 47.1 & 67.8 & 69.6 & $\overline{75.6}$ & 80.0 & 89.7 \\
\hline With DMAEMA & 79.5 & 100.0 & 102.8 & 111.1 & 85.9 & 98.2 \\
\hline
\end{tabular}

Table 4. Crosslink density dependence on the average of the CBB partition coefficient, $\Phi$, as determined for each mol\% TEGDA but derived from a range of evaluated starting concentrations $1-6 \mathrm{wt} \%$ of $\mathrm{CBB}$ at $37^{\circ} \mathrm{C}$.

\begin{tabular}{|l|l|l|l|l|l|l|l|}
\hline $\begin{array}{l}\text { Mol } \\
\text { TEGDA }\end{array}$ & $1 \mathrm{~mol} \%$ & $3 \mathrm{~mol} \%$ & $5 \mathrm{~mol} \%$ & $7 \mathrm{~mol} \%$ & $9 \mathrm{~mol} \%$ & $12 \mathrm{~mol} \%$ & Average \\
\hline $\begin{array}{l}\text { Partition } \\
\text { Coefficient, (\$) }\end{array}$ & 6.54 & 6.62 & 6.72 & 6.81 & 6.59 & 6.84 & $6.69 \pm 0.12$ \\
\hline
\end{tabular}




\section{List of Figures}

Figure 1. A 2-D rendering that schematically illustrates the concept of swelling of a hydrogel by the imbibition of water and schematically illustrates $M_{c}$ and $\xi$ in the 2-D network.

Figure 2. Schematic illustration of the transport of molecular constituents from one phase to another; A. From Liquid 1 to immiscible Liquid 2. B. From Liquid 1 to pseudo-solid wherein molecule-medium complexation phenomena may influence distribution.

Figure 3. A. Schematic illustration of the polymerized molecular constituents of the bioactive hydrogel. B. Structure of CBB. C. Structure of HEPES (4-(2-hydroxyethyl)-1-piperazineethanesulfonic acid).

Figure 4A. The gravimetrically determined degree of hydration $(\%)$ of the p(HEMA)-based hydrogels measured in DI water at $37^{\circ} \mathrm{C}$ as a function of mol \% TEGDA cross-linker when the hydrogel contains $0 \mathrm{~mol} \%$ or 5 mol\% DMAEMA $(n=6)$.

Figure 4B. The gravimetrically determined degree of hydration $(\%)$ of the p(HEMA)-based hydrogels measured in HEPES buffer at $37{ }^{\circ} \mathrm{C}$ as a function of mol \% TEGDA cross-linker when the hydrogel contains 0 mol\% or 5 mol\% DMAEMA $(n=6)$.

Figure 5. Endothermic curves of swollen hydrogels synthesized using 3mol\% TEGDA with and without 5 mol\% DMAEMA.

Figure 6. Partition coefficient, $\Phi$, of CBB for non-DMAEMA containing hydrogels of varying crosslink density (1-12 mol\% TEGDA) as determined in a CBB/HEPES solution of $6.0 \mathrm{wt} \% \mathrm{CBB}$ at $37{ }^{\circ} \mathrm{C}$. 


\section{Graphical Abstract}

PARTITIONING OF COOMASSIE BRILLIANT BLUE INTO DMAEMA CONTAINING POLY(HEMA)-BASED HYDROGELS

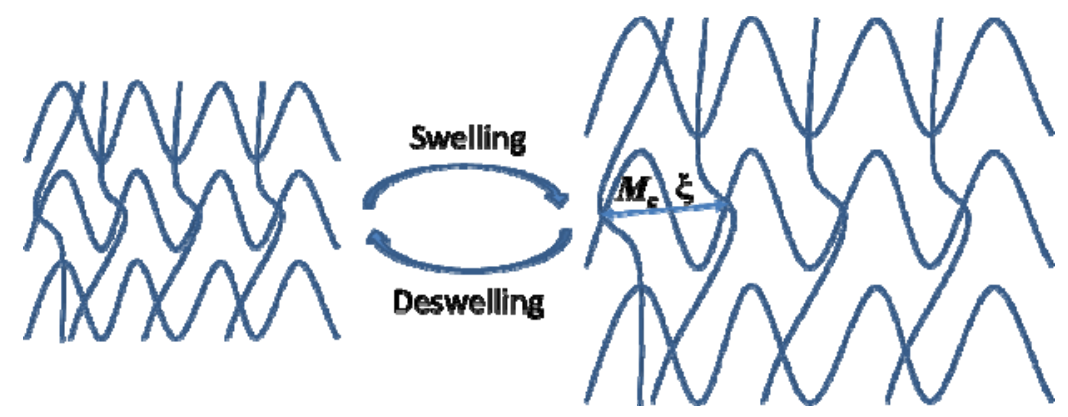


Figure 7. Partition coefficient of CBB for non-DMAEMA containing hydrogels of varying crosslink density (1-12 mol\% TEGDA) as determined in CBB/HEPES solutions of varying initial concentrations $(0.1-6.0 \mathrm{wt} \% \mathrm{CBB})$ at $37^{\circ} \mathrm{C}$.

Figure 8. Partition coefficient of CBB for a hydrogel of 3 mol\% TEGDA with or without $5 \mathrm{~mol} \%$ DMAEMA obtained in a CBB/HEPES solution of $6.0 \mathrm{wt} \% \mathrm{CBB}$ at $37^{\circ} \mathrm{C}$. 